\title{
Renal biopsy in diabetic patients: Histopathological and clinical correlations
}

\author{
Joana Paixão $0^{1, *}$ (D) , Ana Carolina Pimenta ${ }^{2, *}$ (D), Luís Rodrigues ${ }^{2,3}$ (D), David Sousa ${ }^{1}$ (iD), Sandra Santos ${ }^{1}$ (iD), Emanuel Ferreira ${ }^{2}$ (iD), \\ Nuno Afonso 2,3 (iD), Fátima Costa² (iD) Vitor Sousa 4 (iD), Jorge Pratas² (iD), Rui Alves 2,3 (iD \\ * Indicates equal contribution \\ 1 Internal Medicine department, Centro Hospitalar e Universitário de Coimbra, Coimbra, Portugal \\ ${ }^{2}$ Nephrology department, Centro Hospitalar e Universitário de Coimbra, Coimbra, Portugal \\ ${ }^{3}$ University Clinic of Nephrology, Faculty of Medicine, University of Coimbra, Portugal \\ ${ }^{4}$ Institute of Anatomical and Molecular Pathology, Faculty of Medicine, University of Coimbra, Coimbra, Portugal
}

\section{ABSTRACT}

Introduction: Diabetes is the leading cause of chronic kidney disease and end-stage kidney disease worldwide. A kidney biopsy in a diabetic patient must be considered when non-diabetic renal disease is suspected, such as in the presence of a rapid decline in renal function or severe unexplained proteinuria. However, the timing and criteria of a biopsy remain controversial in these patients. We aimed to identify clinical and histological markers that could help differentiate diabetic and non-diabetic renal disease and decide if this invasive approach is needed or not.

Subjects and Methods: We reviewed 30 years of biopsies from diabetic patients performed at a tertiary hospital. We collected patient demographic data, biopsy indications, histological findings, and clinical and analytical data both at the moment of the biopsy and extensive followup. Based on kidney biopsy findings, patients were categorized as isolated diabetic nephropathy, non-diabetic kidney disease, or non-diabetic kidney disease superimposed on diabetic nephropathy (diabetic kidney disease).

Results and Discussion: We enrolled 92 patients, mostly with type 2 diabetes, with a mean age of $62.9 \pm 13.2$ years. Nearly half of them had isolated diabetic nephropathy (53.3\%), and $15.2 \%$ had diabetic nephropathy superimposed on non-diabetic kidney disease, comprising a total of 63 patients (68.5\%) with diabetic kidney disease. Twenty-nine patients (31.5\%) were considered to have non-diabetic kidney disease. These last patients were significantly less likely to need insulin therapy $(p=0.002)$, had more frequently an acute deterioration of renal function ( $p=0.01)$, lower albumin levels $(p=0.03)$, and a higher prevalence of microhematuria $(p=0.001)$. We found the latter to be an independent predictor of non-diabetic kidney disease. Further, patients with the primary diagnosis of diabetic nephropathy had higher survival than those who had nondiabetic kidney disease, contradicting published data.

Conclusions: The criteria for performing a biopsy in diabetic patients still lack consensus, although the priority to identify non-diabetic kidney disease prevails. We believe the non-diabetic kidney disease predictors we describe may prove helpful for determining the need for a histological assessment in diabetic patients.

Key-words: Renal biopsy, diabetes mellitus, diabetic nephropathy, diabetic kidney disease, non-diabetic kidney disease

(C) 2021 Portuguese Journal of Nephrology \& Hypertension. Published by Publicações Ciência \& Vida This is an open access article under the CC BY-NC-ND license (http://creativecommons.org/licenses/by-nc-nd/4.0/).

\section{INTRODUCTION}

Diabetes mellitus (DM) is one of the most serious health problems worldwide, reaching epidemic proportions, mainly due to the rapidly rising global prevalence of type 2 DM. ${ }^{1,2}$ Chronic kidney disease (CKD) is a common complication of DM, which further contributes to cardiovascular risk and mortality. ${ }^{3-6}$ Nowadays, it is commonly accepted that diabetic kidney disease (DKD), traditionally called diabetic nephropathy (DN), is a clinical and pathologically heterogeneous disease, affecting approximately 20 to $40 \%$ of diabetics, type 1 or 2 , and accounting for roughly $45 \%$ of patients on renal replacement therapy. ${ }^{7}$ It no longer indicates a specific pathological phenotype and is mainly a clinical diagnosis, based upon the presence of albuminuria $(>30 \mathrm{mg} /$ $\mathrm{dl}$ ), and/or decreased estimated glomerular filtration rate (eGFR), or both, in a diabetic patient. 8,9

The etiology of DKD is multifactorial. The magnitude and duration of hyperglycemia and hypertension are of utmost significance, in addition to genetic and environmental factors. ${ }^{10}$ Other determinants that increase the probability of DKD or accelerate its development are glomerular hyperfiltration, smoking, obesity, sedentarism, dyslipidemia, proteinuria and high fat and carbohydrates diet. ${ }^{11}$ Concerning pathophysiology, DKD results from a metabolic and hemodynamic impairment, promoting inflammation, endothelial dysfunction, oxidative stress, fibrosis and glomerular hyperfiltration. All these events lead to kidney lesions and potentiate cardiovascular events. ${ }^{12}$ 
Although in type $1 \mathrm{DM}$, with five or more years duration, DN is likely the cause of DKD, particularly if associated with diabetic retinopathy (DR), in type $2 \mathrm{DM}$, this correlation can range widely, as the prevalence of concomitant non-diabetic kidney disease (NDKD) increases. ${ }^{13-15}$ In these cases, only a kidney biopsy (KBx) can accurately settle if DKD is due to DN. Furthermore, albuminuria is no longer required to make a clinical diagnosis of DKD. A substantial minority of diabetic patients and decreased eGFR, with less than $30 \mathrm{mg} / \mathrm{g}$ of albuminuria, have histopathological findings consistent with DKD. Moreover, the regression from moderately increased albuminuria to normoalbuminuria is common in type $1 \mathrm{DM}$ and, although at lower rates, can also occur in type 2 DM. ${ }^{16,17}$

On the other hand, a presumptive diagnosis of DKD should be avoided if there are features present that raise suspicion of an alternative diagnosis for the kidney disease, namely, severely elevated albuminuria ( $>300 \mathrm{mg} / \mathrm{g}$ ) within five years of onset of type $1 \mathrm{DM}$, or many years before type 2 DM diagnosis; red blood cell casts or dysmorphic red blood cells in the urinary sediment; the presence of a systemic disease with renal involvement (e.g. systemic lupus erythematous) or a sudden or atypical increase albuminuria or eGFR decrease. In these patients, a KBx should usually be performed ${ }^{18-19}$

The histological findings consistent with $\mathrm{DN}$ are closely dependent on each health center's biopsy policy. ${ }^{20,21} \mathrm{KBx}$ indication can range from a "restricted" policy to only patients with a suspected alternative diagnosis or an "unrestricted" approach to any diabetic patient who presents with severe albuminuria, low eGFR, or hematuria. One study, as an example, examined biopsies from patients where an alternative diagnosis was suspected. Of those, $29 \%$ had classic diabetic glomerulopathy alone; conversely, with an "unrestricted" approach, 33\% had an alternate diagnosis besides DKD, and another one-third showed only NDKD. ${ }^{22}$ The most common diagnosis of NDKD reported in recent literature were acute tubular necrosis (ATN), immune-mediated glomerular diseases, hypertensive nephrosclerosis and focal segmental glomerulosclerosis (FGSF). ${ }^{23}$

Patients with type $1 \mathrm{DM}$ predominantly develop classical diabetic glomerulopathy, whereas type 2 diabetics, particularly those without albuminuria, may have a myriad of pathological findings. ${ }^{24}$ The earliest recognized pathological abnormality is the thickening of the glomerular basement membrane, which can occur as early as two years after the diagnosis in type $1 \mathrm{DM}$. It is followed by mesangial expansion and nodular (Kimmelstiel-Wilson nodules) or diffuse glomerular sclerosis. ${ }^{25}$ Arteriolar hyalinosis and arteriosclerosis of larger vessels are common, likely representing the combined effect of hyperglycaemia and hypertension. Tubulointerstitial fibrosis anticipates the progression to advanced CKD and end-stage kidney disease (ESKD). As already mentioned, the histological abnormalities in type $2 \mathrm{DM}$ are more heterogeneous, with a more severe vascular and tubulointerstitial disease, most likely translating the distinct phenotypes of these patients. ${ }^{26}$

Several studies have tried to establish clinical hints to differentiate DKD from NDKD. The most cited include DR, the duration of DM, proteinuria, and hematuria. Unfortunately, none has attained enough sensitivity or specificity to dismiss a $\mathrm{KBx} .27,28$
In the present study, the authors aimed to study clinical or laboratory factors predictive of DKD or NDKD in diabetic patients, establishing, whenever possible, correlations with histological features.

\section{SUBJECTS AND METHODS}

We conducted a retrospective study in diabetic patients submitted to a native kidney biopsy (KBx) at the Nephrology Department of Centro Hospitalar e Universitário de Coimbra (CHUC), between January 1990 and December 2020. Electronic medical records were reviewed to collect demographic and clinical data concerning age, gender, time since DM diagnosis, time of death, coexistent comorbidities (DR, hypertension, obesity), and their pharmacological treatment, namely use of angiotensin-converting enzyme inhibitors (ACEi), angiotensin receptor blockers (ARBs), insulin or oral antidiabetic drugs. Laboratory data gathered included serum creatinine, serum albumin, glycated hemoglobin (HbA1c) levels, urinary sediment, spot urine protein/ creatinine ratio (UPCR), and 24-hour collection proteinuria. For followup purposes, we considered serum creatinine levels at the time of $\mathrm{KBx}$ and at one, three, and five years after the procedure. eGFR was estimated based on the Modification of Diet in Renal Disease (MDRD) equation study.

The indications for KBx in our cohort included the following criteria: proteinuria in the nephrotic range ( $>3.5 \mathrm{~g} /$ day) or CKD in the absence of diabetic retinopathy; nephrotic proteinuria or CKD within five years of onset of DM; isolated nephrotic proteinuria; nephrotic syndrome (proteinuria $>3.5 \mathrm{~g} /$ day associated to edema and/or serum albumin $<3.5 \mathrm{~g} / \mathrm{dL}$, with or without hematuria); unexplained microscopic hematuria (presence of five or more red blood cells per high power field in urine analysis), acute kidney injury (AKI) (increase in serum creatinine by $\geq 0.3 \mathrm{mg} / \mathrm{dL}$ within $48 \mathrm{~h}$ or $\geq 1.5$ times baseline within seven days) and an unexplained rapidly worsening renal function in patients previously stable.

We reviewed histopathological reports of those patients and considered diffuse or nodular mesangial expansion, thickening of glomerular basement membranes, and nodular glomerulosclerosis as markers of DN and organized these findings according to the classification outlined by Tervaert et al. ${ }^{25}$ Tubulointerstitial lesions, interstitial inflammation, arteriolar hyalinosis, and diffuse linear staining for IgG along tubular basement membranes and glomerular capillaries were also considered supportive diagnostic features. Based on KBx findings, patients were categorized as isolated DN, isolated NDKD, or DKD (NDKD superimposed on DN).

Statistical analysis was performed using and SPSS ${ }^{\circledR}$ software (version 20). Normal distributed continuous variables were presented as mean \pm standard deviation ( $\mathrm{sd}$ ). Categorical data are shown as numbers and frequencies (percent, \%). We used T-student, chisquared, or Fisher's exact tests to compare differences between groups whenever pertinent. Univariable and multivariable logistic regression models were used to explore risk factors for NDKD. Survival analysis was estimated by the Kaplan-Meier method. The differences between DKD and NDKD groups were assessed using the log-rank test. We considered a two-sided $\alpha$ of less than 0.05 as statistically significant. 


\section{RESULTS AND DISCUSSION}

We analysed data from 96 diabetic patients who underwent a KBx at our hospital between 1990 and 2020. Four patients were excluded due to an insufficient histological sample to establish a diagnosis. The mean age of our final population was $62.9 \pm 13.2$ years, ranging between 21 and 89 years. KBx was performed, on average, $9.5 \pm 8.2$ years after the diagnosis of DM. Only $71.7 \%$ of the patients had previous ophthalmological screening examinations, of whom $33.3 \%$ had DR, including all the five type $1 \mathrm{DM}$ patients (5.2\%). Hypertension and obesity were known in $84.8 \%$ and $30.4 \%$, respectively. Table I summarizes demographic and clinical data for the whole sample.

A quarter of our patients was biopsied for nephrotic syndrome ( $n=23,25 \%)$ or AKI $(n=23,25 \%)$, with the most frequent indication for renal biopsy being proteinuria $(n=29,31.5 \%)$ (Figure 1). Based on histopathological findings, nearly half of them had isolated DN ( $n=49,53.3 \%)$, and $14(15.2 \%)$ had DN superimposed on NDKD, comprising a total of 63 patients (68.5\%) with DKD. Using the Tervaert pathological classification, nodular sclerosis and advanced diabetic glomerulosclerosis (classes III and IV) were more frequently observed in the isolated DN group (85.7\%). In comparison, mild to severe mesangial expansion (class II) was more prevalent in DN superimposed on NDKD patients (68.3\%). Twenty-nine patients (31.5\%) were considered to have NDKD. All type 1 diabetic patients were included in the DN group. Besides DN, ATN was the main histological final

\section{Table I}

Demographic and clinical characteristics of diabetes mellitus patients with kidney biopsy

\begin{tabular}{|c|c|c|}
\hline \multicolumn{3}{|c|}{$\begin{array}{l}\text { Demographic and clinical characteristics of diabetes mellitus (DM) patients } \\
\text { with kidney biopsy (KBX). }\end{array}$} \\
\hline & & Min-Max \\
\hline Age (years), mean \pm sd & $62.9 \pm 13.2$ & $21-89$ \\
\hline Male gender, $\mathrm{n}(\%)$ & $63(68.5)$ & \\
\hline Time since DM diagnosis (years), mean \pm sd & $9.5 \pm 8.2$ & $0.0-45.0$ \\
\hline HbA1c (\%), mean \pm sd & $7.4 \pm 1.7$ & $5.5-15.0$ \\
\hline Diabetic retinopathy, $\mathrm{n}(\%)$ & $22(23.9)$ & \\
\hline Obesity, n (\%) & $28(30.4)$ & \\
\hline Hypertension, n (\%) & $78(84.8)$ & \\
\hline Microhematuria ( $\geq 5 \mathrm{RBC} /$ field), $\mathrm{n}(\%)$ & $23(25)$ & \\
\hline Proteinuria (g), mean \pm sd & $6.4 \pm 5.2$ & $0.1-22.7$ \\
\hline Nephrotic proteinuria, $n(\%)$ & $54(58.7)$ & \\
\hline Serum albumin $(\mathrm{g})$, mean $\pm \mathrm{sd}$ & $3.3 \pm 0.9$ & $1.6-6.6$ \\
\hline \multicolumn{3}{|l|}{ Pharmacological treatment } \\
\hline Insulin, n (\%) & $32(34.8)$ & \\
\hline Oral antidiabetic drugs, $n(\%)$ & $44(47.8)$ & \\
\hline Insulin + oral antidiabetic drugs, n (\%) & $15(16.3)$ & \\
\hline ACEi, $n(\%)$ & $36(39.1)$ & \\
\hline ARBs, $n(\%)$ & $14(15.2)$ & \\
\hline ACEi + ARBs, n (\%) & $4(4.3)$ & \\
\hline \multicolumn{3}{|l|}{ GFR (ml/min/1,73m2, MDRD), mean $\pm \mathrm{sd}$} \\
\hline At the time of the kidney biopsy & $37.1 \pm 31.6$ & $2.4-139.8$ \\
\hline 1 year after kidney biopsy & $48.5 \pm 29.8$ & $8.1-161.8$ \\
\hline 3 years after kidney biopsy & $51.3 \pm 28.9$ & $12.3-110.5$ \\
\hline 5 years after kidney biopsy & $47.9 \pm 33.1$ & $7.3-118.7$ \\
\hline $\begin{array}{l}\text { Life expectancy after kidney biopsy (years), } \\
\text { mean } \pm \text { sd }\end{array}$ & $6.8 \pm 7.5$ & $0.0-34.1$ \\
\hline
\end{tabular}

\section{Figure 1}

Indications for renal biopsy in diabetic patients of the present study

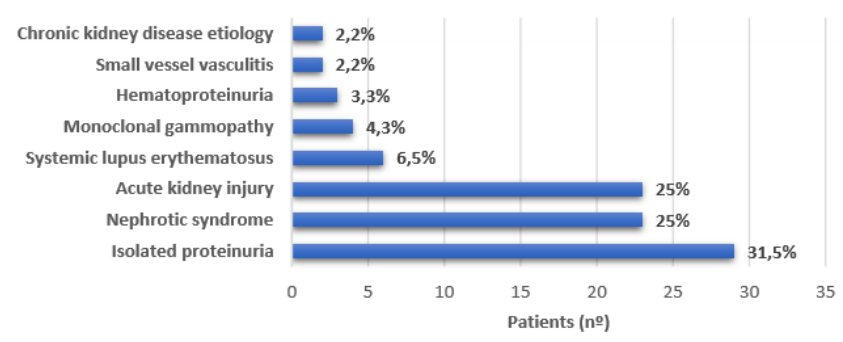

\section{Figure 2}

List and number of the main pathological diagnosis of the present study

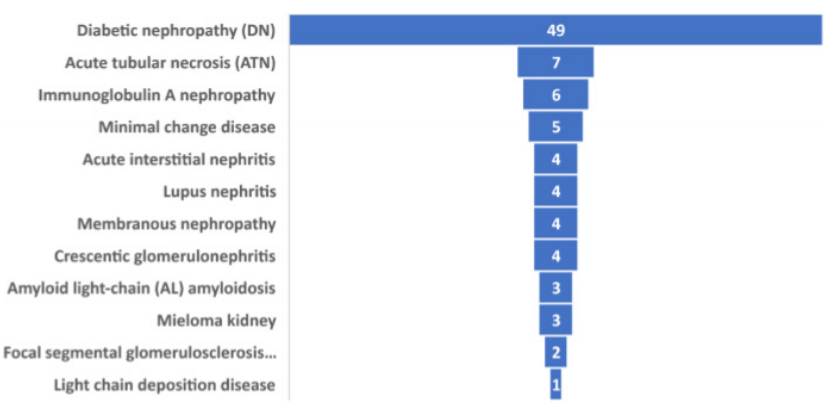

diagnosis ( $n=7,7.6 \%)$, closely followed by IgA nephropathy $(n=6$, $6.5 \%)$ and minimal change disease $(n=5,5.4 \%)$. All the pathological diagnoses are listed in Figure 2.

When KBx were performed, patients with NDKD were less likely to need insulin therapy ( $24.1 \%$ vs. $62.5 \%, p=0.002)$, had lower albumin levels ( 2.9 vs. $3.4 \mathrm{mg} / \mathrm{dL}, \mathrm{p}=0.03)$, and a higher prevalence of microhematuria ( $41.9 \%$ vs. $10.4 \%, p=0.001)$. We found the latter to be an independent predictor of NDKD; despite ATN accounting for $7.6 \%$ $(n=7)$ of this group, the majority of patients with microhematuria had glomerulopathies.

For all the cohorts, the mean eGFR was $37.1 \pm 31.6 \mathrm{ml} / \mathrm{min} / 1.73$ $\mathrm{m}^{2}$ at the time of $\mathrm{KBx}$ and $47.9 \mathrm{ml} \pm 33.1 \mathrm{ml} / \mathrm{min} / 1,73 \mathrm{~m}^{2}$ at the fifth year of follow-up. This improvement of eGFR over time is clearly related to the significant percentage of patients with AKI in our population. However, we found a significant negative association between the rate of decline of eGFR and the presence of histological DKD, with NDKD patients evincing a greater loss of renal function five years after $\mathrm{KBx}(\mathrm{R} 2-0.339, \mathrm{p}=0.004)$. Likewise, patients presenting with acute deterioration of renal function were significantly more prevalent among the NDKD group (60.9\% vs. $30.4 \%, p=0.01)$.

When analysing survival data, we also found that NDKD patients had a significantly lower global life expectancy compared to DKD patients ( $8.4 \pm 1.2$ vs. $16.6 \pm 2.4)(p=0.03)$ (Figure 3). 


\section{Figure 3}

Kaplan-Meier estimates of survival according in the patients with DN and NDKD as the primary pathological diagnosis on $\mathrm{KBx}$

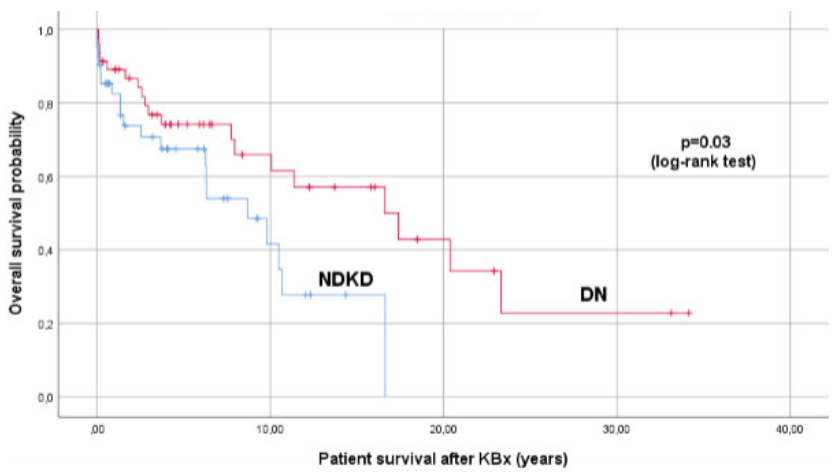

\section{DISCUSSION}

The role of a $\mathrm{KBx}$ in $\mathrm{DM}$ patients remains controversial and the criteria to perform it lacks consensus. Besides the potential use for DN-related investigational purposes, this invasive procedure in diabetic patients seems mainly helpful in excluding NDKD. In accordance with previously published series, our patients were submitted to KBx when the clinical and laboratory findings raised the suspicion of conditions other than DKD. Severe or rapid onset proteinuria, especially if less than five years from the diagnosis of type $1 \mathrm{DM}$ or in the absence of concomitant $\mathrm{DR}$, was the most common indication for requiring a $\mathrm{KBx}$ in our sample. The coexistence of other systemic diseases with wellknown renal involvement, the evidence of hematuria or AKI were also frequent criteria. This heterogeneity of indications in daily medical practice highly explains the assorted prevalence of NDKD in clinical studies, with values ranging from $6.5 \%$ to $94 \%{ }^{29}$ In our series, DN was excluded in almost a third of the patients studied, all with type 2 DM, bringing a global prevalence of NDKD of $31.5 \%$.

Several diagnoses had been reported in diabetic patients with NDKD, with significant heterogeneity in the histological patterns pictured among different populations. A systematic analysis by Fiorentino and colleagues concluded that IgA nephropathy was the most common renal pathology overall, mainly in Asia, with a higher frequency of FGSF described in European studies. ${ }^{18}$ In our analysis, ATN was an important histological finding, the leading diagnosis after classic DN. Similarly, an American study by Sharma et al. concluded that ATN was the most common NDKD, found alone or alongside DN lesions in $28.4 \%$ of those patients. ${ }^{22}$ These results highlight the acknowledged susceptibility of diabetic patients to ischemia and tubulointerstitial damage and are of clinical relevance, since ATN is associated with a higher risk of progression towards ESKD, especially in the setting of the reduced renal reserve associated with $\mathrm{DM}^{22,32}$

Certain factors, such as microscopic hematuria (especially with active urinary sediment), severe or sudden-onset proteinuria, the absence of diabetic retinopathy, a short duration of DM, low $\mathrm{HbA1c}$ levels, and acute deterioration of renal function have been described as clinical predictors of NDKD. ${ }^{29,30}$ Accordingly, we found microhematuria and kidney function impairment, either acutely or progressive, to be more common among the NDKD group.

Whereas the need for insulin therapy has been frequently associated with DN, its absence was considered a strong predictor of NDKD among 80 Croatian diabetic patients studied by Horvatic et al. ${ }^{29,31}$ In our analysis, insulin independence was also a distinctive marker of NDKD, probably reflecting a more accurate metabolic control in this subgroup of patients where DM was not the predominant pathology. Finally, low albumin levels prevailed among histopathological NDKD, which might be related to the significant proportion of patients biopsied for albumin-wasting events, such as in nephrotic syndrome cases or inflammatory conditions, as those found in AKI patients.

The presence and extent of the pathological lesions assessed by $\mathrm{KBx}$ in diabetic patients have proved its prognostic value, with isolated or mixed forms of DKD meaning higher chances of progression to ESKD. ${ }^{26}$ In our cohort, NDKD was associated with a significantly lower life expectancy when compared to DKD, a different result from the one recently reported by Bermejo et al., who inferred that DN was an independent risk factor for mortality. ${ }^{27}$ Our results further disagree with other data published reporting better renal and overall survival of NDKD when compared to DKD. ${ }^{28,29}$ The severity of comorbidities diagnosed among our patients, some of them with systemic involvement, may partially explain the poorest survival in the NDKD cohort.

There are many limitations in our study. The small number of patients and a monocentric, retrospective design with a relatively short follow-up period may explain the paucity of clinically significant predictors of NDKD found among our cohort. Similar to other studies, there was also a significant selection bias related to the KBx criteria, raising the risk of overestimating the actual prevalence of NDKD in patients with DM. However, to our knowledge, this is the first study characterizing patterns of renal disease among the Portuguese diabetic population, as others focused only on patients with clinical suspicion of NDRD, ${ }^{32}$ acquiring strength in the urge of gathering multicentric data and consensual approaches in order to provide the best renal care to our patients.

In conclusion, KBx remains the gold standard approach for diagnosis, treatment decisions and outcome prediction in patients with kidney diseases. However, currently, there are no standardized criteria or consensus about mandatory indications nor clinical usefulness in patients with DM. Therefore, the decision to perform a kidney biopsy in these patients should be based on clinical judgment and health centers' policies. In our cohort, a significant proportion of patients yielded an NDKD and, therefore, was potentially worthy of receiving targeted therapies to improve kidney function. The evidence of an acute deterioration of renal function, microhematuria, low albumin levels and absence of insulin therapy were independent predictors of NDKD, suggesting their potential use for determining the histological assessment in those patients. However, the decision to perform a KBx in DM should be individualized and based on careful clinical judgment to promote our patients' best management, ensuring early diagnosis, particularly of NDKD, and timely treatment, thus avoiding irreversible sequelae, and improving renal and overall prognosis.

Disclosure of potential conflicts of interest: none declared. 


\section{References}

1. Zimmet PZ, Magliano DJ, Herman WH, et al. Diabetes: a 21st century challenge. Lancet Diab End 2014;2(1):56-64

2. Ogurtsova $K$, da Rocha Fernandes JD, Huang $\mathrm{Y}$, et al. IDF diabetes atlas: global estimates for the prevalence of diabetes for 2015 and 2040. Diabetes Res Clin Pract 2017;128:40-50

3. Rawshani A, Rawshani A, Franzen S, et al. Mortality and cardiovascular disease in type 1 and type 2 diabetes. N Engl J Med 2017;376(15):1407-1418

4. Thomas B, Matsushita $\mathrm{K}$, Abate $\mathrm{KH}$, et al. Global cardiovascular and renal outcomes of reduced GFR. J Am Soc Nephrol 2017;28(7):2167-2179

5. Chang YT, Wu JL, Hsu CC, et al. Diabetes and end-stage renal disease synergistically contribute to increased incidence of cardiovascular events: a nationwide follow-up study during 1998-2009. Diabetes Care 2014:37(1):277-285

6. Afkarian M, Sachs MC, Kestenbaum B, et al. Kidney disease and increased mortality risk in type 2 diabetes. J Am Soc Nephrol 2013;24(2):302-308

7. Afkarian M, Zelnick LR, Hall YN, et al. Clinical manifestations of kidney disease among US adults with diabetes, 1988-2014. JAMA 2016;316(6):602-610

8. Tonneijck L, Muskiet MH, Smits MM, et al. Glomerular hyperfiltration in diabetes: mechanisms, clinical significance, and treatment. J Am Soc Nephrol 2017;28(4):1023-1039

9. Oi C, Mao X, Zhang Z et al. Classification and differential diagnosis of diabetic nephropathy. J Diabetes Res 2017;2017:8637138

10. Tan J, Zwi L, Marshall MR et al. Presentation, pathology and prognosis of renal disease in type 2 diabetes. BMJ Open Diabetes Research and Care 2017: 11:5(1):e000412

11. Hojs R, Ekart R, Bevc $S$ et al. Markers of inflammation and oxidative stress in the development and progression of renal disease in diabetic patients. Nephron 2016;133-159

12. Pichler R, Afkarian M, Dieter BP et al. Immunity and inflammation in diabetic kidney disease: translating mechanisms to biomarkers and treatment strategies. Am J Physiol 2017; 312:F716

13. Wolf $\mathrm{G}$, Muller N, Mandecka A, et al. Association of diabetic retinopathy and renal function in patients with types 1 and 2 diabetes mellitus. Clin Nephrol 2007;68(2):81-86

14. De Boer IH, AfkarianM, Rue TC, et al. Renal outcomes in patients with type 1 diabetes and macroalbuminuria. J Am Soc Nephrol 2014; 25:2342

15. Olsen S, Mogensen CE. How often is NIDDM complicated with non-diabetic renal disease? An analysis of renal biopsies and the literature. Diabetologia 1996;39(12):1638-1645

16. Perkins BA, Ficociello LH, Silva KH, et al. Regression of microalbuminuria in type 1 diabetes. $\mathrm{N}$ Eng J Med 2003;348(23):2285-2293

17. Bentata $\mathrm{Y}$, Karimi I, Benabdellah $\mathrm{N}$, et al. Albuminuria in type 2 diabetes mellitus: from remission to progression. Ren Fail 2016:38(3):481-483

18. Fiorentino M, Bolignano D, Tesar V et al. Renal biopsy in patients with diabetes: A pooled metaanalysis of 48 studies. Nephrol Dial Transplant 2017;32:97-110

19. Perkovic $\mathrm{V}$, Agarwal $\mathrm{R}$, Fioretto $\mathrm{P}$, et al. Management of patients with diabetes and CKD: conclusions from a "Kidney Disease: Improving Global Outcomes" (KDIGO) controversies conference. Kidney Int 2016;90(6):1175-1183

20. Zhuo L, Ren W, Li W, et al. Evaluation of renal biopsies in type 2 diabetic patients with kidney disease: a clinicopathological study of 216 cases. Int Urol Nephrol 2013;45(1):173-179

21. Mazzucco G, Bertani T, Fortunato $M$, et al. Different patterns of renal damage in type 2 diabetes mellitus: a multicentric study on 393 biopsies. Am J Kidney Dis 2002;39(4):713-720

22. Sharma SG, Bomback AS, Radhakrishnan J, et al. The modern spectrum of renal biopsy findings in patients with diabetes. Clin J Am Soc Nephrol 2013;8(10):1718-1724

23. Toyoda M, Najafian B, Kim Y, et al. Podocyte detachment and reduced glomerular capillary endothelial fenestration in human type 1 diabetic nephropathy. Diabetes. 2007;56(8):2155-2160
24. Alicic RZ, Rooney MT, Tuttle KR. Diabetic kidney disease: challenges, progress, and possibilities. Clin J Am Soc Nephrol 2017;12:2032-45

25. Tervaert TW, Mooyaart AL, Amann K, et al. Pathologic classification of diabetic nephropathy. J Am Soc Nephrol 2010;21(4):556-563

26. Santoro D, Torreggiani M, Pellicanò V, et al. Kidney Biopsy in Type 2 Diabetic Patients: Critical Reflections on Present Indications and Diagnostic Alternatives Int J Mol Sci 2021; 22(11):5425

27. Bermejo $S$, González $E$, López-Revuelta $K$, et al. Risk factors for non-diabetic renal disease in diabetic patients, Clin Kidney J 2020;13,3:380-388

28. Tong X, Yu Q, Ankawi, G. et al. Insights into the Role of Renal Biopsy in Patients with T2DM: A Literature Review of Global Renal Biopsy Results. Diabetes Ther 2020; 11: 1983-1999

29. García-Martín F, González Monte E, Hernández Martínez E, et al. When to perform renal biopsy in patients with type2 diabetes mellitus? Predictive model of non-diabetic renal disease. Nefrologia (Engl Ed) 2020;40(2):180-189

30. Fontana F, Perrone R, Giaroni F, et al. Clinical Predictors of Nondiabetic Kidney Disease in Patients with Diabetes: A Single-Center Study", Int J Neph 2021, Article ID 9999621, https://doi. org/10.1155/2021/9999621

31. Horvatic I, Tisljar M, Kacinari P, et al. Non-diabetic renal disease in Croatian patients with type 2 diabetes mellitus. Diabetes Res Clin Pract 2014;104(3):443-50

32. Dias JM, Alves RV, Góis M, et al. Prevalence, Etiology and Clinical Characteristics of Biopsy Proven Non-Diabetic Renal Disease in a Population of 67 Diabetic Patients. J Clin Nephrol Ren Care 2019; 5:046

\section{ORCID}

Joana Paixão (iD) 0000-0002-2050-405X

Ana Carolina Pimenta (iD 0000-0003-3992-0763

Luis Rodrigues (iD) 0000-0003-0122-982X

David Sousa (iD) 0000-0003-4815-3981

Sandra Santos (iD) 0000-0002-1871-3696

Emanuel Ferreira (ID) 0000-0003-1320-2894

Nuno Afonso (iD) 0000-0002-4112-9236

Fátima Costa iD 0000-0002-5654-3157

Vitor Sousa iD 0000-0003-3219-1078

Jorge Pratas (iD) 0000-0002-1977-4802

Rui Alves (iD) 0000-0003-3922-3618

Joana Paixão, MD

Serviço de Medicina Interna, Centro Hospitalar e Universitário de Coimbra Praceta Prof. Mota Pinto, 3004-561 Coimbra, Portugal

E-mail: jipaixao13@gmail.com 